\title{
Nonreciprocal Oersted field contribution to the current-induced frequency shift of magnetostatic surface waves
}

\author{
Mohammad Haidar" and Matthieu Bailleul ${ }^{\dagger}$ \\ Institut de Physique et Chimie des Matériaux de Strasbourg - IPCMS and NIE, UMR 7504 CNRS - Université de Strasbourg, \\ 23 rue du Loess, BP 43, 67034 Strasbourg Cedex 2, France
}

Mikhail Kostylev

School of Physics, The University of Western Australia, Crawley, WA 6009, Australia

Yuyang Lao

University of Science and Technology, Hefei, China and School of Physics, The University of Western Australia, Crawley, WA 6009, Australia

(Received 18 October 2013; revised manuscript received 11 February 2014; published 31 March 2014)

\begin{abstract}
The influence of an electrical current on the propagation of magnetostatic surface waves is investigated in a relatively thick $(40 \mathrm{~nm})$ permalloy film both experimentally and theoretically. Contrary to previously studied thinner films where the dominating effect is the current-induced spin-wave Doppler shift, the magnetic field generated by the current (Oersted field) is found to induce a strong nonreciprocal frequency shift which overcompensates the Doppler shift. The measured current-induced frequency shift is in agreement with the developed theory. The theory relates the sign of the frequency shift to the spin-wave modal profiles. The good agreement between the experiment and the theory confirms a recent prediction of a counterintuitive mode localization for magnetostatic surface waves in the dipole-exchange regime.
\end{abstract}

DOI: 10.1103/PhysRevB.89.094426

PACS number(s): 75.30.Ds, 75.76.+j, 75.78.-n, 76.50.+g

\section{INTRODUCTION}

The spin waves are the elementary magnetic excitations of ferromagnets. Although they are known for a long time, their study at the nanometer scale in thin films is the subject of a recent field of research called magnonics [1], which proposes to use them as information vectors for future applications in data storage and signal processing [2-4]. Another field of research where spin waves play an important role is that of spin-transfer torque, through the phenomenon of the current-induced spin-wave Doppler shift (CISWDS): When an electrical current flows along a metal ferromagnet in which a spin wave is excited, there is a transfer of angular momentum along the spin-wave propagation direction, which shifts the spin-wave frequency by an amount proportional to the degree of spin polarization of the current [5]. The CISWDS can therefore be used to probe directly spin-polarized electron transport in various experimental conditions and materials [6-11]. It was also suggested that another spin-torque effect (the current-induced modification of the spin-wave attenuation) could be used to amplify them $[9,11,12]$. It is essential to understand precisely the influence of the electrical current onto the propagation of the spin wave in order to be able to rule out possible concurrent physical effects which are likely to combine with the spin-transfer torque, in particular the effect of the inhomogeneous magnetic field generated directly by the electrical current (the Oersted field). For future development in these two fields (magnonics and spin-wave spin-transfer torque) a good understanding of the fundamental physics of

\footnotetext{
*Present address: Physics Department, University of Gothenburg, 412 96, Gothenburg, Sweden; mohammad.haidar@hotmail.fr

†'matthieu.bailleul@ipcms.unistra.fr
}

spin-wave propagation in metallic ferromagnetic films and of the influence of a dc electrical current on it is therefore needed.

The most relevant configuration for experimental studies of spin-wave propagation is the so-called magnetostatic surface wave (MSSW) configuration (also known as Damon-Eshbach configuration) in which the equilibrium magnetization $\mathbf{M}$ and the spin-wave wave vector $\mathbf{k}$ are perpendicular to each other, and both lie in the plane of the film $[13,14]$. This configuration has two advantages: (i) because $\mathbf{M}$ is oriented in the film plane, moderate magnetic fields are sufficient to magnetize the film, and (ii) because $\mathbf{M}$ is perpendicular to $\mathbf{k}$, and lies in the film plane, the precession of magnetization induces two components of the dynamic demagnetizing field: in the film plane and perpendicular to it, both with a strong dependence on $|\mathbf{k}|$. This unique structure of the dynamic demagnetizing field translates into a relatively high group velocity. Due to the large group velocity, for a given relaxation time, these waves propagate quite far before they completely die off due to the attenuation in the medium. This significantly facilitates their measurements with respect to the other spin-wave configurations. However, MSSW also has a very specific property called nonreciprocity: the amplitudes, mode profiles, and frequencies of the waves traveling in the two opposite propagation directions $(k>0$ and $k<0$ ) do not coincide. The amplitude nonreciprocity is a property related to MSSW excitation by external energy sources: the efficiency of excitation of spin waves by a microstrip or coplanar inductive antenna located on the film surfaces is larger for one propagation direction than for the opposite one [15]. The modal-profile nonreciprocity manifests itself in the fact that these spin waves have a larger amplitude on one side of the film than on the other one (surface character of the wave). The surface at which the wave is localized swaps upon reversal of the propagation direction [13]. Finally, frequency 
nonreciprocity may also be present whenever the film is asymmetric in the thickness direction [16]. The nonreciprocity of MSSW had been studied in much detail in thick, low magnetization yttrium iron garnet (YIG) films $[13,15]$. For thin, high magnetization permalloy (Py) films used in most magnonic studies, MSSW nonreciprocities were first observed by Brillouin light scattering (see, e.g., Ref. [17] and the detailed discussion in Ref. [18]) and investigated later with more detail using spin-wave antennas techniques [16,19-21]. Quite recently it has been shown theoretically that for a given applied-field direction, MSSW in thin Py films may be localized at the film surface opposite to the one of MSSW localization in thick YIG films due to the more pronounced role of the exchange interaction in the magnetization dynamics in the Py films [18].

In the context of the studies of the current-induced spinwave Doppler shift, the amplitude and frequency MSSW nonreciprocities complicate the extraction of the Doppler shift, because signals corresponding to counterpropagating spin waves cannot be directly compared contrary to the first CISWDS measurement which dealt with reciprocal spin waves [5]. Different procedures have been proposed to extract the Doppler shift, either by combining measurements taken at different polarities of $M$ and $k$ [6], or by combining measurements taken at different polarities of $I$ and $k$ [10].

In this paper we build upon these previous works. We measure very precisely the nonreciprocity of propagation of magnetostatic surface waves and its modification by an electrical current in a permalloy film which is thicker $(40 \mathrm{~nm})$ than the ones employed in the previous studies $(6-20 \mathrm{~nm}$ in Refs. $[6,10,11])$. Surprisingly we observe that the currentinduced frequency shift behaves very differently from what is observed for the thinner films: it does not scale linearly with the wave vector and can even change its sign. We attribute this behavior to a large nonreciprocal contribution from the Oersted field of the dc current which combines with the Doppler effect. To observe a noticeable Doppler frequency shift, large densities of dc current are required. For the same large current density the total current through a thicker film is larger, which results in a larger Oersted field than for a thinner film. Furthermore, for the same wave number the mode-profile asymmetry is also larger for a thicker film, so that the contribution of the Oersted field to the mode frequency becomes non-negligible with respect to the Doppler frequency shift. Our study also reveals that the Oersted field and Doppler contributions have the opposite signs. It is impossible to distinguish between the two contributions by employing symmetry considerations because they behave in the same way as functions of the directions of $\mathbf{I}, \mathbf{k}$, and H. On the other hand, as we show here, they have different dependencies on the magnitude of the wave vector. The total frequency shifts calculated using the dipole-exchange theory are in good agreement with the measured ones. Because the nonreciprocal Oersted contribution to the current-induced frequency shift is very sensitive to the mode profile asymmetry, our observation provides a confirmation of the counterintuitive MSSW localization behavior predicted in Ref. [18].

The paper is organized as follows. The experimental results are presented in Sec. II. In Sec. III we provide a qualitative interpretation of the measured current-induced frequency shifts. The theoretical calculations are presented in Sec. IV and we conclude in Sec. V.

\section{EXPERIMENTAL RESULTS}

\section{A. Propagating spin-wave measurements for $I=0$}

The sample used in the experiment consists of a 40-nmthick permalloy (Py) film sandwiched between $\mathrm{Al}_{2} \mathrm{O}_{3}$ layers. It was grown on an intrinsic silicon substrate by magnetron sputtering. The chip contains several devices of the type shown in Fig. 1(a). Each device comprises a Py strip of width $w$ and a pair of narrow-band microwave spin-wave antennae of meander shape for the excitation and detection of spin waves with wave vector $k$. The antennae are separated from the strip by a 120 -nm-thick SiOx insulating layer. In addition, four dc pads are connected to the strip in order to launch a dc current $I$ into it and to measure its resistance. Figures 1(b)-1(d) show scanning electron microscopy images of each device. In each panel we indicate the strip width and the characteristic wave vector for each device. Spin waves are excited by the antenna with a main excitation peak centered at a wave vector $k_{M}$ and a secondary peak centered at a lower wave vector $k_{S}$ as described in the Appendix of Ref. [22]. The samples are placed in a uniform static magnetic field $\mathbf{H}$ applied in the film plane, perpendicular to the propagation direction of the spin waves, which corresponds to the magnetostatic surface wave configuration. The propagating spin-wave spectroscopy (PSWS) measurements are performed as described in detail elsewhere [10,22,23].

The devices were first characterized in the absence of the dc current. Figure 2(a) shows typical mutual inductance signals: $\Delta L_{21}$ (solid curve) which corresponds to a wave propagating

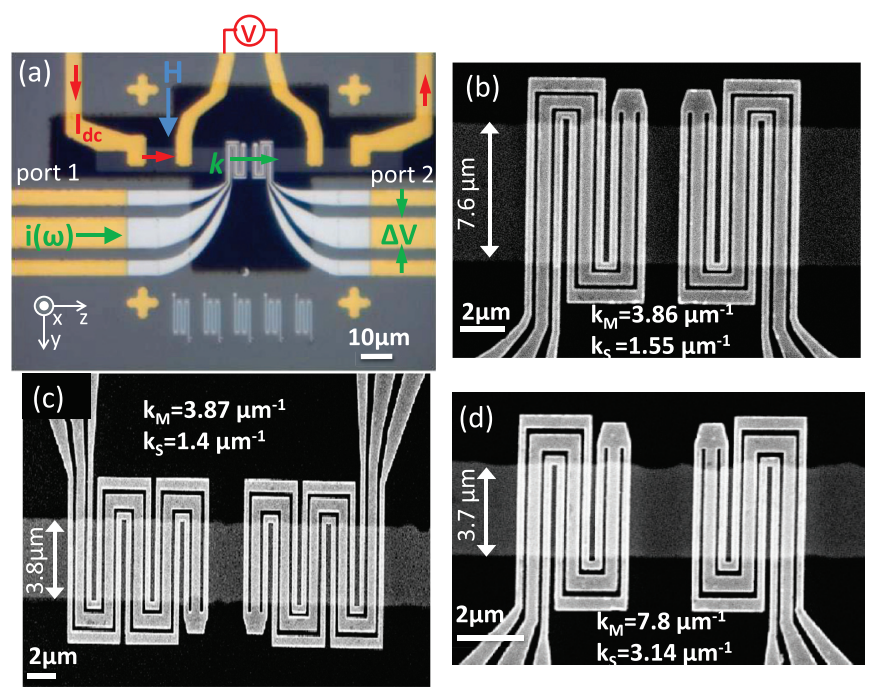

FIG. 1. (Color online) (a) Optical microscope image of one CISWDS device. One recognizes the permalloy strip ion milled from a continuous film $\left(\mathrm{Al}_{2} \mathrm{O}_{3} 21 \mathrm{~nm} / \mathrm{Py} 40 \mathrm{~nm} / \mathrm{Al}_{2} \mathrm{O}_{3} 5 \mathrm{~nm}\right)$, the four $\mathrm{dc}$ current pads and the two coplanar waveguides (Ti $10 \mathrm{~nm} / \mathrm{Au}$ $60 \mathrm{~nm}$ ), the insulating spacer ( $\mathrm{SiOx} 120 \mathrm{~nm}$ ), and the two spin-wave antennae (Ti $10 \mathrm{~nm} / \mathrm{Al} 120 \mathrm{~nm}$ ). The conventions used in the text for the directions of positive $\mathbf{k}, \mathbf{I}$, and $\mathbf{H}$ are shown. (b)-(d) Scanning electron microscope images showing the strip and the antennae for each of the three fabricated devices. 

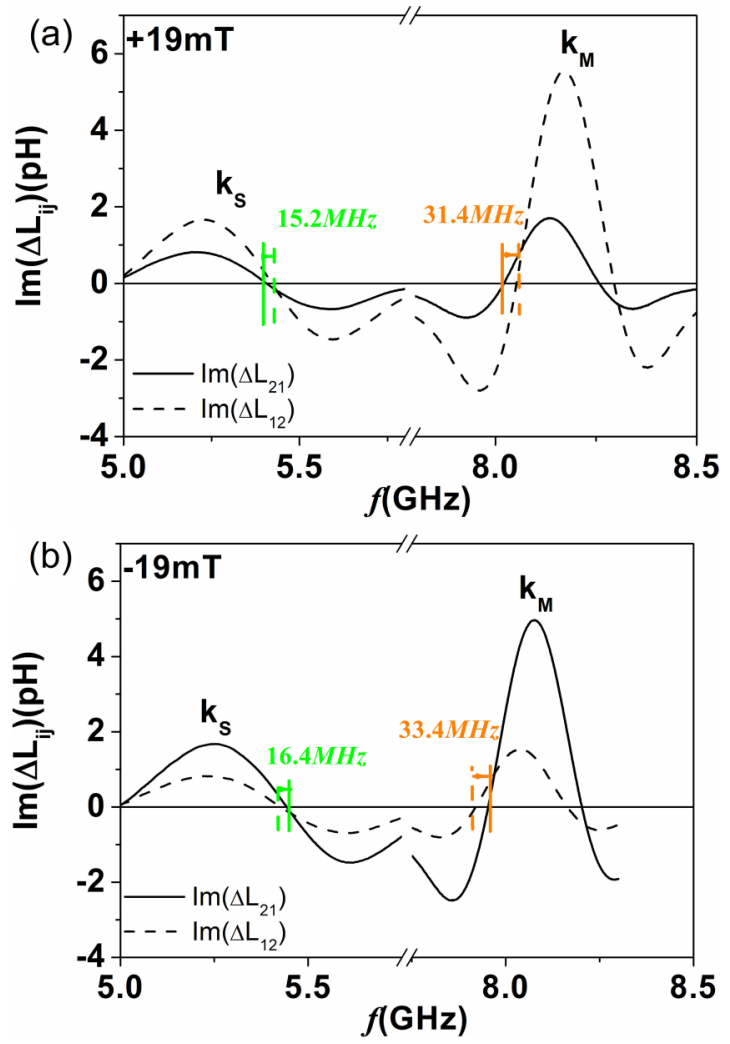

FIG. 2. (Color online) The imaginary parts of the mutual inductance signals for $k>0$ and $k<0$ propagations (solid and dashed curves, respectively). The signals were measured for the device shown in Fig. 1(c). The strip width is $w=3.8 \mu \mathrm{m}$ and the characteristic wave numbers are $k_{M}=3.86 \mu \mathrm{m}^{-1}$ and $k_{S}=1.4 \mu \mathrm{m}^{-1}$. The measurements were performed in an external field (a) $\mu_{0} H=19 \mathrm{mT}$ and (b) $\mu_{0} H=-19 \mathrm{mT}$ in the absence of the dc current.

from port 1 to port $2[k>0$, see notations in Fig. 1(a)] and $\Delta L_{12}$ (dashed curve) which corresponds to a wave propagating from port 2 to port $1(k<0)$. For both signals we observe two distinct wave packets centered at 5.4 and $8.1 \mathrm{GHz}$ at an external field of $19 \mathrm{mT}$. These frequencies are in good agreement with the values expected from the MSSW dispersion relation with wave vectors $k_{M}$ and $k_{S}$, respectively [13]. One also notices that the transmitted amplitude for $k<0$ is higher than the one for $k>0$ for both peaks. The ratio of the amplitude of the $k<0$ signal to the $k>0$ signal is about 3 and 2 for the $k_{M}$ and $k_{S}$ peaks, respectively. This amplitude asymmetry is in agreement with Refs. [15,20,21], where it was explained based on differences in elliptical polarizations of the oscillating magnetization of the spin wave and of the microwave field generated by the antennae. The spin wave whose magnetization precession has the same polarization as the driving microwave field is excited more strongly than the spin wave with the opposite polarization. In agreement with the theoretical expectation [24], the wave with higher amplitude is propagating with a wave vector $\mathbf{k} \|(\mathbf{n} \times \mathbf{M})$, where $\mathbf{n}$ is the internal normal to the film surface close to which the antenna is located, which corresponds in our experiment to the $k<0$ signal for the $+H$ field orientation. We also observe that the $k<0$ signal lies at a slightly higher frequency than the $k>0$ signal. The frequency shift is about 31.4 and $15.2 \mathrm{MHz}$ for $k_{M}$ and $k_{S}$ peaks, respectively. As in the case of thinner films [10], we attribute this frequency nonreciprocity to the combination of the modal-profile nonreciprocity with some asymmetry of the magnetic properties of the films with respect to its midplane (e.g., a different surface anisotropy at the top and bottom interfaces [25] or an inhomogeneous magnetization distribution across the film thickness [26,27]). A quantitative interpretation of this feature is left for future work. As we switch the direction of the static field to the negative one, we observe that the $k>0$ and $k<0$ signals swap their amplitudes and frequencies, which means that the $k>0$ signal now has a higher amplitude and a higher frequency with respect to the $k<0$ signal, as shown in Fig. 2(b). Hence, the amplitude and frequency nonreciprocities reverse when the direction of the external field is reversed [28]. This is in good agreement with the interpretation given above because both the polarization of the oscillating magnetization and the modal-profile asymmetry are expected to reverse when the equilibrium magnetization is switched but not the polarization of the microwave field of the antennae.

\section{B. Current-induced modifications of the spin-wave signals}

Let us now investigate the effect of an electrical current on the propagating spin waves. Figure 3(a) shows the mutualinductance spectra recorded in the presence of an electrical current $I= \pm 7.5 \mathrm{~mA}$. The small current-induced frequency shifts are better seen in Fig. 3(b) which shows a zoom close to the intercept of the curves with the horizontal axis. The $+I$ curves (blue lines) appear to be at slightly smaller frequencies than the $-I$ curves (red lines) and the shift is clearly higher for $\Delta L_{21}$ (solid curve). As in Ref. [10], we define $\delta f_{i j}=f_{i j}(+I)-f_{i j}(-I)$, where $f_{i j}(I)$ is the frequency at which the $\operatorname{Im} \Delta L_{i j}(I)$ signal $(i, j=1,2)$ vanishes. One obtains $\delta f_{12}=-2.1 \mathrm{MHz}$ and $\delta f_{21}=-5.7 \mathrm{MHz}$. These two values are combined as follows: $\delta f_{\text {even }}=\left(\delta f_{12}+\delta f_{21}\right) / 4=$ $-1.95 \mathrm{MHz}$ is the part of the current-induced frequency shift which is even in $k$ and $\delta f_{\text {odd }}=\left(\delta f_{12}-\delta f_{21}\right) / 4=+0.9 \mathrm{MHz}$ is the part of the current-induced frequency shift which is odd in $k$. Figures 3(c) and 3(d) show the PSWS signals measured when the direction of the magnetic field is switched. The current-induced frequency shifts are now $\delta f_{21}=+2.6 \mathrm{MHz}$ and $\delta f_{12}=+6.2 \mathrm{MHz}$, which gives $\delta f_{\text {odd }}=+0.9 \mathrm{MHz}$ and $\delta f_{\text {even }}=+2.2 \mathrm{MHz}$. Apparently the part of the currentinduced frequency shift that is even in $k$ is also odd in $H$, and the part which is odd in $k$ is even in $H$. The current-induced frequency shifts also scale linearly with the dc current. This is exemplified in Fig. 4(a) for $\delta f_{\text {odd }}$.

Before we further discuss $\delta f_{\text {odd }}$, which is the part that contains the CISWDS (the Doppler shift changes sign between two counterpropagating spin waves) and also the nonreciprocal Oersted field contribution we will discuss below, let us briefly discuss $\delta f_{\text {even }}$. As for thinner films [10], we attribute it to a (reciprocal) Oersted field contribution induced by a top/bottom asymmetry of the ferromagnetic metal film: If the electrical properties are not perfectly symmetric with respect to the film midplane (e.g., the top part is slightly more conductive than the bottom part), the Oersted field is not entirely antisymmetric with respect to the film midplane and does not average out to zero, so that a small residual field will add to or subtract 
(a)
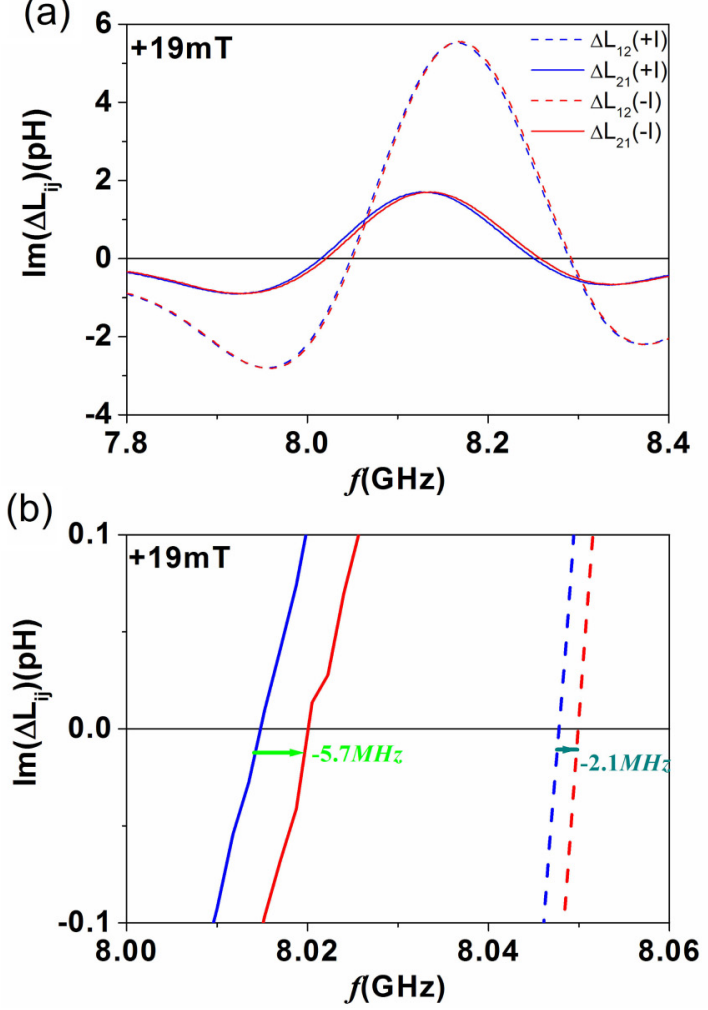

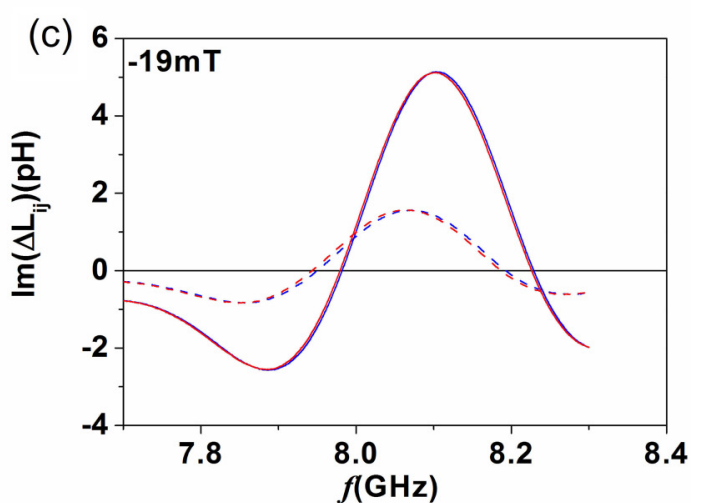

(d)

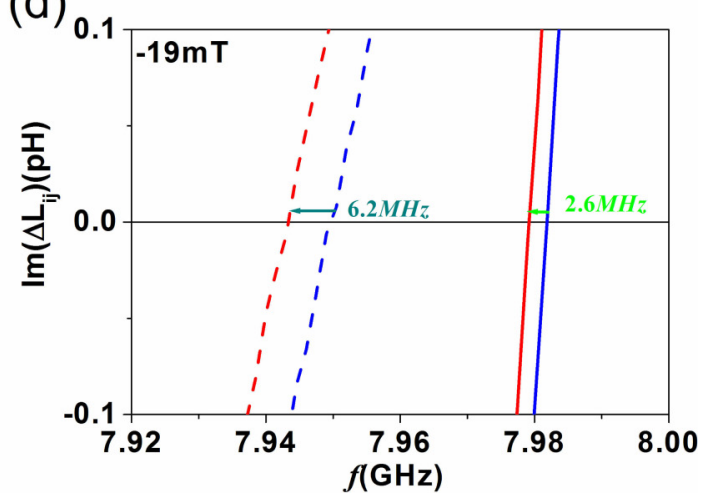

FIG. 3. (Color online) The mutual inductance signals measured in the presence of the dc current. The measurements were performed at (a) $+19 \mathrm{mT}$ and (c) $-19 \mathrm{mT}$ at $|I|=7.5 \mathrm{~mA}$ for the same device as Fig. 2. (b) and (d) Zoomed views of the signals showing the current-induced frequency shifts.

from the applied magnetic field and therefore modify the frequency (see, e.g., Sec. 5 in the Supplementary Material of Ref. [11]). Similarly, if the magnetic properties of the film are not perfectly symmetric (e.g. magnetization pinning is stronger at the top than at the bottom surface), there might be a slight (reciprocal) asymmetry of the spin-wave profile with respect to the sample midplane, so that a perfectly antisymmetric Oersted field weighted by this profile would not average out to zero. In this picture the asymmetry occurring across the film thickness originates from the film itself, it is therefore not expected to reverse when $k$ is reversed. These effects are thus expected to be reciprocal and to lead to a current-induced frequency shift even in $k$. Because the Oersted field combines vectorially with the external field $H$, this contribution is also expected to be odd in $H$, as it was deduced from Figs. 3(b) and 3(d). A quantitative understanding of this even contribution is beyond the scope of this paper.

Let us now focus on the part of the current-induced frequency shift which is odd in $k$. The current-induced spinwave Doppler shift $\Delta f_{\text {Dop }}$ expected to contribute to this part writes [5]

$$
\Delta f_{\text {Dop }}=-\frac{\mu_{B}}{2 \pi|e|} \frac{P}{M_{s}} \frac{I}{L w} k,
$$

where $w$ and $L$ are the width and the thickness of the ferromagnetic metal strip, $\mu_{B}$ is the Bohr magnetron, $|e|$ is the magnitude of the electron charge, $M_{S}$ is the saturation magnetization, and $P=\frac{J_{\uparrow}-J_{\downarrow}}{J_{\uparrow}+J_{\downarrow}}$ is the degree of the spin polarization of the electrical current. To explore the wave vector dependence of $\delta f_{\text {odd }}$, we compare the current-induced frequency shifts measured for the three devices (main excitation peaks at $k_{M}=3.9$ and $7.8 \mu \mathrm{m}^{-1}$ ) and we also use the current-induced shifts measured for the secondary peaks $\left(k_{S}=1.4\right.$ to $\left.3.14 \mu \mathrm{m}^{-1}\right)$. To account for the difference in the strip widths of the devices, $\delta f_{\text {odd }}$ is actually plotted as a function of the current density $J=I / L w$. The slopes of the linear fits obtained in each case are plotted in Fig. 4(b) as a function of the wave vector, together with the data points obtained following the same procedure for a $10 \mathrm{~nm}$ film. The difference between the two film thicknesses is obvious. For the $10 \mathrm{~nm}$ film, $\delta f_{\text {odd }}$ scales linearly with the wave vector, as expected from the Doppler effect [see Eq. (1)]. On the other hand, for the $40 \mathrm{~nm}$ film, $\delta f_{\text {odd }}$ first increases between $k=1.4$ and $3.4 \mu \mathrm{m}^{-1}$, then saturates and finally strongly drops to become negative at $k=7.8 \mu \mathrm{m}^{-1}$. A direct application of Eq. (1) leads to the following evaluates for the degree of spin polarization $P$ : $0.52 \pm 0.02$ for $L=10 \mathrm{~nm}$, a value which can be understood by considering the spin-polarized electron scattering processes acting in a permalloy thin film [10], and between 0.73 and -0.24 for $L=40 \mathrm{~nm}$ (the two values corresponding to $k=1.4$ and $7.8 \mu \mathrm{m}^{-1}$, respectively). We believe this latter range of values does not make sense: There is no reason for the degree of spin polarization to depend on the wave vector in this range (the spin-wave wavelength $\lambda=0.8-4.5 \mu \mathrm{m}$ remains much larger than any of the characteristic lengths for the electrical transport in such a film) and there is no reason for it to become negative (the dominant electron scattering processes at large thickness is the scattering by the alloy disorder, which is known to give 

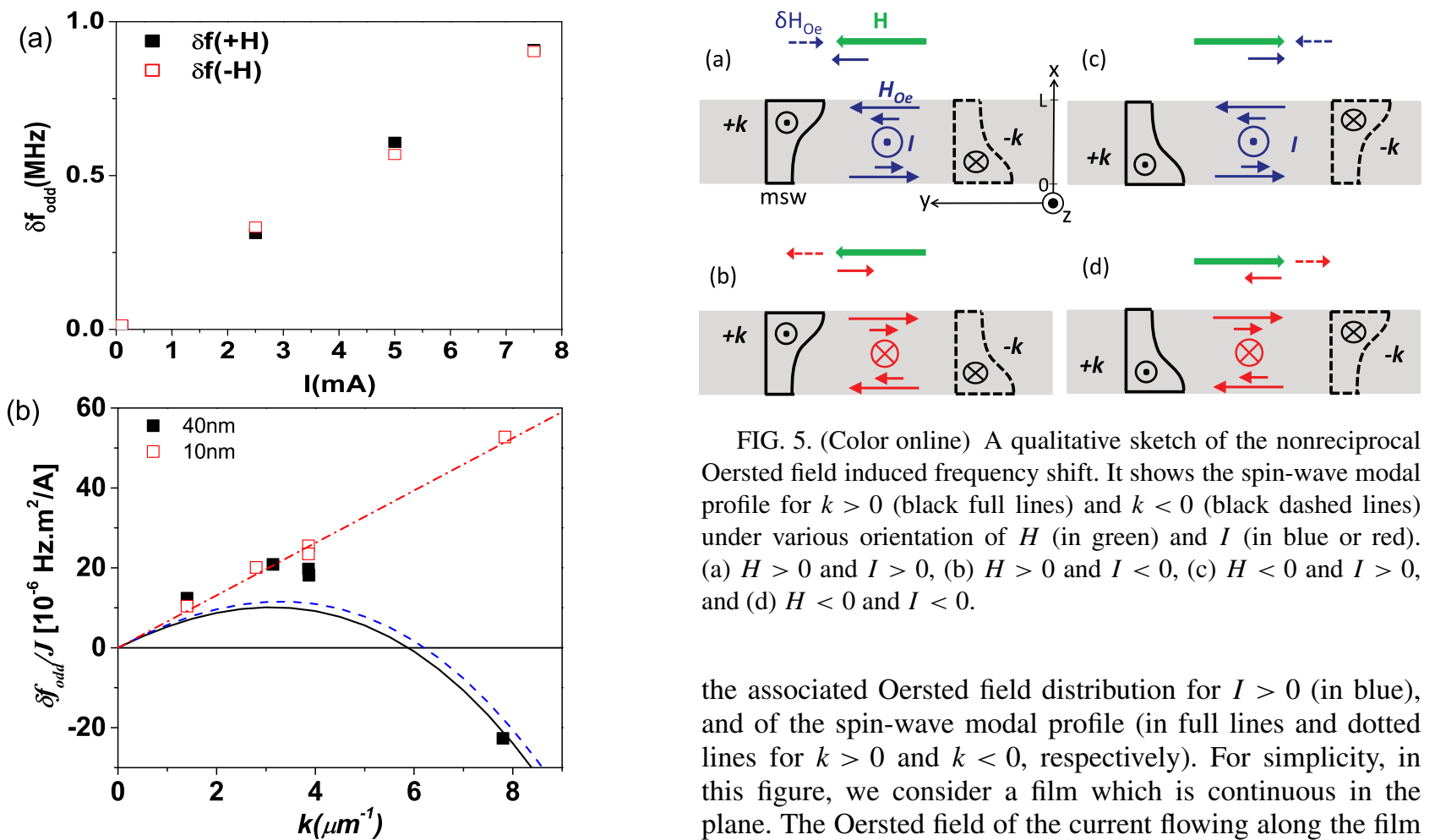

FIG. 5. (Color online) A qualitative sketch of the nonreciprocal Oersted field induced frequency shift. It shows the spin-wave modal profile for $k>0$ (black full lines) and $k<0$ (black dashed lines) under various orientation of $H$ (in green) and $I$ (in blue or red). (a) $H>0$ and $I>0$, (b) $H>0$ and $I<0$, (c) $H<0$ and $I>0$, and (d) $H<0$ and $I<0$.

FIG. 4. (Color online) (a) Variation of $\delta f_{\text {odd }}$ as a function of the electrical current (same device as in Figs. 2 and 3). (b) Ratio of $\delta f_{\text {odd }}$ to the current density $J$ as a function of the wave vector for $40 \mathrm{~nm}$ (filled squares) and $10 \mathrm{~nm}$ (open squares) thin films. Lines: Respective theoretical data. Solid black line: Rigorous numerical calculation in real space for the 40-nm-thick film. Dashed blue line: The same, but analytical solution using Eq. (21). Dash-dotted red line: Film thickness is $10 \mathrm{~nm}$ (for this thickness, the results of both rigorous numerical calculation and analytical formula coincide to graphical accuracy with a linear dependence corresponding to the CISWDS only).

rise to a strong positive spin polarization [29]). Therefore, we believe that another effect combines with the CISWDS to generate the $\delta f_{\text {odd }}$ we measure.

\section{QUALITATIVE EXPLANATION}

In this section we present a naive qualitative picture which explains the experimentally observed nonlinear wave-number dependence of the current-induced frequency shift which translates into the unphysical wave-number dependence of the extracted degree of spin polarization together with its unphysical change of sign. We claim that the wave-number dependence of the frequency shift is due to one more process taking place in parallel to the Doppler effect. This is the "Oersted field induced frequency shift" (OFIFS). It was previously mentioned in Ref. [9], but has not been explored in detail yet. The idea is that the Oersted field generated by the dc current $H_{\mathrm{Oe}}$ can modify the spin-wave dispersion in a metallic ferromagnetic film and make the spin-wave dispersion nonreciprocal.

This effect is illustrated in Fig. 5(a), which shows a sketch of the strip cross section (in gray), of the electrical current and

the associated Oersted field distribution for $I>0$ (in blue), and of the spin-wave modal profile (in full lines and dotted lines for $k>0$ and $k<0$, respectively). For simplicity, in this figure, we consider a film which is continuous in the plane. The Oersted field of the current flowing along the film is antisymmetric across the film thickness: it varies linearly across the thickness and has two maxima (a positive and a negative) at the two opposite film surfaces. Due to this contribution, the total static magnetic field is nonuniform across the film thickness. From Fig. 5(a) it is clear that the Oersted field suitably weighted by the spin-wave modal profile does not average out to zero but to a finite value defined as $\delta H_{\mathrm{Oe}}$ in the figure. For $k>0$ the resultant field adds to the external field, so that the frequency is increased, whereas for $k<0$ it subtracts from $H$ so that the frequency is decreased. Naturally the effect reverses when $I$ is reversed [see Fig. 5(b)]. The effect is therefore odd in $I$ and $k$, similar to CISWDS [see Eq. (1)]. Figures 5(c) and 5(d) illustrate the situation when $\mathbf{H}$ is reversed. In that case, the modal-profile asymmetry is reversed, and the resultant Oersted field reverses accordingly. However, because the static magnetic field points now in the opposite direction, the magnitudes of the total field are the same as in Figs. 5(a) and 5(b). Consequently, the nonreciprocal Oersted field contribution to the spin-wave frequencies does not change upon the reversal of $\mathbf{H}$, similar to the CISWDS contribution. From this discussion, it is clear that in order for the Oersted field induced frequency nonreciprocity to appear, the waves should possess asymmetry of modal profiles across the film thickness together with modal-profile nonreciprocity, i.e., the profile asymmetry should be different for the waves propagating in the two opposite directions. At this point of the discussion, it is important to notice that the sign of the OFIFS depends on the sign of the modal-profile nonreciprocity. We have chosen in Fig. 5 a sign of the modal-profile nonreciprocity such that the resultant Oersted field induced frequency shift is opposite to the CISWDS expected for a positive spin polarization (in that case the Doppler effect shifts the frequency up when the spin-wave phase velocity is co-aligned with the electron flow, i.e., when it is anti-aligned to the current). This sign of modal-profile nonreciprocity is compatible with our 
experimental result, which can be explained if a concurrent process compensates the Doppler frequency shift.

Let us now discuss the origin of this modal-profile nonreciprocity. As a first approach, one is tempted to resort to the standard Damon-Eshbach (DE) picture of magnetostatic waves. Neglecting the exchange interaction, one obtains a wave with a surface character [14]: The profile of the dynamic magnetization across the film thickness (in the direction $x$ ) is a combination of two exponentials $[\exp (-k x)$ and $\exp (k x)]$, with an asymmetry between the two components such that the amplitude of magnetization precession is maximum at one of the film surfaces. The problem with this approach is the sign of the nonreciprocity. Indeed, in the DE picture the surface at which the wave has more amplitude is the one whose internal normal $\mathbf{n}$ verifies [30]

$$
\mathbf{k} /|k|=\mathbf{n} \times \mathbf{M} /|\mathbf{M}| .
$$

One can easily convince oneself that this rule actually provides a modal-profile nonreciprocity opposite to what is sketched in Fig. 5 [31]. Therefore, in the DE picture the OFIFS is expected to have the same sign as the Doppler frequency shift and to increase the total value of the shift, which is not compatible with our experimental result. This contradiction is removed if the exchange interaction is taken into account. In that case the sign of the modal-profile nonreciprocity is determined not only by the sign of $k$ [as follows from Eq. (2)], but also by the magnitude of $k$ [18] such that in a broad range of wave numbers the spin wave is characterized by the modal profiles shown in Fig. 5. In the next section we give a full theoretical calculation of the OFIFS for this situation. For a methodological purpose we also provide in the Appendix a full derivation of the OFIFS in the DE picture. Although this theory is not directly applicable to our experiment, it shows in a very transparent way how the Oersted field can be introduced

$$
\omega \mathbf{m}_{k}=\mid \begin{gathered}
-\left[\omega_{H}+\gamma H_{\mathrm{Oe}}+\omega_{M}\left(-\alpha \partial^{2} / \partial x^{2}+\alpha k^{2}+1 / 2\right)\right] \delta \\
\omega_{M}\left(G_{q}-G_{p}+\delta / 2\right)
\end{gathered}
$$

In these equations $H_{\mathrm{Oe}}=J_{0} J(x-L / 2)$, where $J$ is measured in $\mathrm{A} \mathrm{cm}^{-2}$ and $J_{0}=4 \pi / 10$ is a factor converting the Oersted field into Gaussian units, $\alpha$ is the exchange constant, $\delta=\delta(s)$ is the Dirac $\delta$ function, $\omega_{H}=\gamma H, \omega_{M}=(H /|H|) \gamma 4 \pi M_{s}$, and $\gamma$ is the gyromagnetic coefficient for the magnetic material. The column vector $\mathbf{m}_{k}$ has now components $\left(m_{k}^{(1)}, m_{k}^{(2)}\right)$. In the form which is the most convenient for the analysis below, the components of the Green's function are presented in Ref. [18]. They are as follows:

$$
\begin{gathered}
G_{p}(s)=\frac{|k|}{2} \exp (-|k||s|), \\
G_{q}(s)=\operatorname{sign}(s) \frac{k}{2} \exp (-|k||s|),
\end{gathered}
$$

where $s=x-x^{\prime}$. The $G_{p}$ component describes the dipole field parallel to the magnetization vector $\mathbf{m}_{k}$ generating it. The $G_{q}$ component is the field component perpendicular to $\mathbf{m}_{k}$. as a perturbation into the equations determining the spin-wave frequencies.

\section{THEORY}

\section{A. Initial equations}

To construct the theory we will use the frame of reference shown in Figs. 1 and 5 and CGS units. The film is assumed to be infinite in the $y$ direction and magnetized to saturation by an external magnetic field $\mathbf{H}=H \mathbf{e}_{y}$ (e $\mathbf{e}_{y}$ is the unit vector in the $y$ direction). The dynamic magnetization vector $\mathbf{m}$ has only two nonvanishing components $\left(m_{x}, m_{z}\right)$, which are perpendicular to the static (equilibrium) magnetization vector $\mathbf{M}=M_{s}(\mathbf{H} /|H|) \mathbf{e}_{y}$. The direction $z$ is in the film plane along the spin-wave wave vector. The dynamic effective field $\mathbf{h}_{\text {eff }}$ which enters Landau-Lifshitz equation [30] has two components: the exchange field $\mathbf{h}_{\mathrm{ex}}$ and the dipole field $\mathbf{h}_{d}$. For the dynamic magnetization and field in the form of a plane spin wave with a wave number $k\left(\mathbf{k}=k \mathbf{e}_{z}\right)$ and a frequency $\omega$ traveling along $z$ we may use

$$
\mathbf{m}, \mathbf{h}_{\mathrm{eff}}=\mathbf{m}_{k}, \mathbf{h}_{\mathrm{eff} k} \exp (i \omega t-i k z) .
$$

We present the dynamic dipole field as a tensor Green's function $\mathbf{G}_{k}$ of dynamic magnetization [32]:

$$
\mathbf{h}_{d k}(x)=\int_{0}^{L} \mathbf{G}_{k}\left(x-x^{\prime}\right) \mathbf{m}_{k}\left(x^{\prime}\right) d x^{\prime}=\mathbf{G}_{k} \otimes \mathbf{m}_{k},
$$

where the symbol $\otimes$ denotes the convolution operation and $L$ is the film thickness. A coordinate transformation

$$
m_{x k}=\left(m_{k}^{(1)}+m_{k}^{(2)}\right) / 2, \quad m_{z k}=\left(m_{k}^{(1)}-m_{k}^{(2)}\right) /(2 i)
$$

and a similar transformation for the components of $h_{\text {eff } k}$ reduce the linearized Landau-Lifshitz equation to a system of integrodifferential equations

$\begin{gathered}\omega_{M}\left(G_{q}+G_{p}-\delta / 2\right) \\ {\left[\omega_{H}+\gamma H_{O e}+\omega_{M}\left(-\alpha \partial^{2} / \partial x^{2}+\alpha k^{2}+1 / 2\right)\right] \delta}\end{gathered} \mid \otimes \mathbf{m}_{k}$.

One sees that the eigenfrequency of spin waves $\omega$ in Eq. (6) represents an eigenvalue of the integrodifferential operator. The terms involving the exchange constant $\alpha$ originate from the exchange contribution to the spin-wave frequency (see Ref. [32] for details).

\section{B. Solution}

The theory is based on solving Eq. (6) using the BoubnovGalerkin method [33]. This method consists of the expansion of $\mathbf{m}_{k}$ in a Fourier series [32]. For simplicity we assume the "unpinned surface spins" exchange boundary conditions on both film surfaces. In this case the system of cosine functions is the natural choice of a full orthonormal basis of functions which satisfy the boundary conditions.

The general form of the dipole exchange spectrum in our applied-field configuration can be characterized as a dipoledominated Damon-Eshbach-like (DE) branch intersected by a number of dispersionless branches of standing spin-wave modes (SSW) (see, e.g., Fig. 1 in Ref. [34]). In the vicinity 
of the intersection points the branches repulse and the respective modal profiles across the film thickness hybridize. In ferromagnetic metallic films with thicknesses of several tens of nanometers and high saturation magnetization, the second SSW branch and all higher order branches are situated well above the upper frequency limit for the existence of the DE branch [35]. As a consequence, only the first SSW may hybridize with the DE-like mode, a situation which is correctly accounted for by retaining only the first two terms of the Fourier-series expansion (see, e.g., Eq. (47) in Ref. [36]):

$$
\mathbf{m}_{k}(x)=\mathbf{m}_{k 0}+\sqrt{2} \mathbf{m}_{k 1} \cos (\pi x / L) .
$$

We substitute this solution into Eq. (6) and project the resultant equation on the orthonormal basis of these cosine functions. As a result we obtain a system of four algebraic equations. The matrix $C_{k}$ of the coefficients of this system of equations has the form as follows:

$$
C_{k}=\left|\begin{array}{cccc}
-A_{00} & -B_{00} & -A_{01} & B_{01} \\
B_{00} & A_{00} & B_{01} & A_{01} \\
-A_{01} & -B_{01} & -A_{11} & -B_{11} \\
-B_{01} & A_{01} & B_{11} & A_{11}
\end{array}\right|,
$$

where $A_{00}=\omega_{H}+\omega_{M} \alpha k^{2}+\omega_{M} / 2, B_{00}=\left[1 / 2-P_{00}\right] \omega_{M}$, $A_{11}=A_{00}+\omega_{M} \alpha(\pi / L)^{2}, \quad B_{11}=\left[1 / 2-P_{11}\right] \omega_{M}, \quad B_{01}=$ $\omega_{M} Q_{01}$, and $A_{01}=\gamma J J_{0} d_{01}$. The quantity $\omega_{H}$ originates from the Zeeman interaction contribution to the total effective field, the term $\omega_{M} / 2$ originates from the out-of-plane thin-film demagnetizing factor, and the terms involving $\alpha$ originate from the exchange interaction. The in-plane effective exchange field scales as a square of the in-plane spin-wave wave number. It gives rise to the term $\omega_{M} \alpha k^{2}$. The out-of-plane effective exchange field contribution, which affects the first SSW is given by $\omega_{M} \alpha(\pi / L)^{2}$.

The quantities $P_{00}, P_{11}$, and $Q_{01}$ are particular cases of the dipole elements $P_{n n^{\prime}}$ and $Q_{n n^{\prime}}$ derived in Ref. [32]. They are obtained by projecting $G_{p}$ and $G_{q}$ [Eqs. (7) and (8), respectively] on the basis of the cosine functions, which gives

$$
\begin{gathered}
P_{00}=1-[1-\exp (-|k| L)] /(|k| L), \\
P_{11}=(k L)^{2}\left[1-2(|k| L) \frac{1+\exp (-|k| L)}{(k L)^{2}+\pi^{2}}\right], \\
Q_{01}=-2 \sqrt{2}(k L) \frac{1+\exp (-|k| L)}{(k L)^{2}+\pi^{2}} .
\end{gathered}
$$

These quantities originate from the dipole-field contributions to the total dynamic effective field. More precisely, the matrix element $P(1-P)$ is an in-plane (out-of-plane) traveling spin-wave counterpart of the in-plane (out-of-plane) diagonal element of the demagnetizing tensor of a thin body which enters the Kittel equation for the in-plane ferromagnetic resonance. From this point of view, they can be regarded as the effective dynamic demagnetizing factors for traveling spin waves. The $Q$ element is the effective antidiagonal demagnetizing factor. This element is responsible for the surface character of the Damon-Eshbach mode and also for the nonreciprocity of the wave propagation, since this is the only element which is odd in $k$. Furthermore, because $G_{q}$ is an antisymmetric function of $s$ [see Eq. (8)], the $Q_{n n^{\prime}}$ elements with even values of $n+n^{\prime}$ vanish. Similarly, the $P_{n n^{\prime}}$ elements with odd values of $n+n^{\prime}$ vanish, because $G_{p}$ is an even function of $s$.

The quantity $d_{01}$ originates from the contribution of the Oersted field generated by the dc current. It is obtained by projecting the thickness dependence of $H_{\mathrm{Oe}}$ onto the same basis of the cosine functions. The Oersted field element reads

$$
d_{01}=-2 \sqrt{2} L / \pi^{2} .
$$

Similar to $G_{q}(s)$, the Oersted field is antisymmetric across the film thickness. Therefore only the $(0,1)$ and $(1,0)$ components of $d$ do not vanish.

The vector-matrix equation

$$
\omega\left|m_{k 0}^{(1)}, m_{k 0}^{(2)}, m_{k 1}^{(1)}, m_{k 1}^{(2)}\right\rangle=C_{k}\left|m_{k 0}^{(1)}, m_{k 0}^{(2)}, m_{k 1}^{(1)}, m_{k 1}^{(2)}\right\rangle
$$

represents the equation of motion (in the frequency space) for the eigenmodes of a ferromagnetic film in the presence of a dc current. Here $|\cdots\rangle$ denotes a column vector composed of the respective components. This equation shows that the eigenfrequencies of spin waves are given by the eigenvalues of $C_{k}$ and the elements of its eigenvectors represent the Fourier amplitudes of the dynamic magnetization profiles [Eq. (9)] for the eigenmodes.

Accordingly, the values of the eigenfrequencies are given by the condition

$$
\operatorname{det}\left(C_{k}-I \omega\right)=0,
$$

where $I$ is the identity matrix.

Evaluating Eq. (16) analytically, one obtains an implicit expression of the dispersion relation in the presence of the $\mathrm{dc}$ current. This relation can be cast in the following form:

$\left(\omega_{1}^{2}-\omega^{2}\right)\left(\omega_{0}^{2}-\omega^{2}\right)+4 \gamma J J_{0} \omega \omega_{M}^{2} d_{01} Q_{01}\left(P_{00}-P_{11}\right)=0$.

In this equation $\omega_{0}$ and $\omega_{1}$ are the (positive) roots of the biquadratic equation which represents the dispersion relation for $J=0$ :

$$
\begin{gathered}
\left(\omega_{11}^{2}-\omega^{2}\right)\left(\omega_{00}^{2}-\omega^{2}\right)-\omega_{M}^{2} Q_{01}^{2}\left[\left(\omega_{11}^{2}-\omega^{2}\right)+\left(\omega_{00}^{2}-\omega^{2}\right)\right] \\
+\omega_{M}^{4}\left[Q_{01}^{4}-Q_{01}^{2}\left(P_{00}-P_{11}\right)^{2}+\alpha^{2}(\pi / L)^{4}\right]=0,
\end{gathered}
$$

and $\omega_{00}\left(\omega_{11}\right)$ is the positive root of the determinant of the upper (lower) $2 \times 2$ diagonal block of the block matrix $C_{k}-I \omega$. These roots are given by the expressions as follows:

$$
\begin{aligned}
\omega_{00}^{2}=\left[\omega_{H}\right. & \left.+\omega_{M}\left(\alpha k^{2}+P_{00}\right)\right]\left[\omega_{H}+\omega_{M}\left(1+\alpha k^{2}-P_{00}\right)\right], \\
\omega_{11}^{2}= & \left(\omega_{H}+\omega_{M}\left\{\alpha\left[k^{2}+(\pi / L)^{2}\right]+P_{11}\right\}\right) \\
& \times\left(\omega_{H}+\omega_{M}\left\{1+\alpha\left[k^{2}+(\pi / L)^{2}\right]-P_{11}\right\}\right) .
\end{aligned}
$$

Note that in order to obtain the dispersion relation in the simple form of Eq. (17) we neglected the terms of the second order in $J$ because $H_{\mathrm{Oe}}^{2} \ll\left(4 \pi M_{s}\right)^{2}$.

The frequency shift due to the presence of the dc current is small compared to the unperturbed spin-wave frequency given by Eq. (18). Therefore we may assume that $\omega=\omega_{0}+\delta \omega$, where $|\delta \omega| \ll \omega_{0}$. This allows one to expand Eq. (17) in Taylor series in $\delta \omega$. Keeping only the linear terms of this expansion 
we obtain a very simple formula for OFIFS [37]:

$$
\delta \omega=\frac{2 \gamma J J_{0} \omega_{M}^{2} d_{01} Q_{01}\left(P_{00}-P_{11}\right)}{\omega_{1}^{2}-\omega_{0}^{2}} .
$$

Let us analyze this expression. $P_{00}$ and $P_{11}$ are positive, $P_{00}>P_{11}$, and $d_{01}$ is negative. The sign of $Q_{01}$ changes upon switching the direction of the wave vector. $Q_{01}$ is negative for $k>0$. The unperturbed dispersion for the case of our sample ( $L=40 \mathrm{~nm}$ ) given by Eq. (18) is shown in Fig. 6(a). For this thickness, the first SSW intersects the DE wave at a relatively high wave number. Hybridization of the DE wave and of the first SSW results in repulsion of the branches. As one sees from this graph, all the wave numbers for which the experimental data were taken correspond to the lower branch of this spectrum. To calculate OFIFS for this branch one has to assume that $\omega_{0}$ is the frequency which corresponds to it and $\omega_{1}$ is the frequency for the upper frequency branch for the same wave number. This assumption implies that $\omega_{0}<\omega_{1}$ and hence $\delta \omega>0$ for $k>0$ and $J>0$. This result is in agreement with our experiment [i.e., the OFIFS is of the opposite sign compared to the Doppler shift for $P>0$, see Eq. (1)].

If we were working at a much higher frequency, we would now assume that $\omega_{0}$ belongs to the upper branch, $\omega_{1}$ is then the respective frequency from the lower branch, and hence $\delta \omega<0$. In this case one recovers the same sign for the OFIFS as in the exchange-free theory (see the Appendix). This can be seen from the argument as follows: For vanishing exchange $(\alpha \rightarrow 0)$, all SSW modes become degenerate, their frequency being equal to the FMR frequency of the film (see, e.g., Fig. 2 in Ref. [34]). As a consequence, the DE mode is now at a frequency higher than all the modes it could hybridize with.

One also notices that the frequency shift scales as $Q_{01}$. As seen from Eqs. (10) and (18) $Q_{01}$ is responsible for the hybridization and repulsion of the DE and first SSW branches. As stated above, it is also responsible for the surface character of the waves as well as for the modal-profile asymmetry and nonreciprocity (all in the absence of the current). More precisely, the modal-profile asymmetry can be characterized by a parameter

$$
\begin{aligned}
S= & {\left[m_{k}^{(2)}(x=0)-m_{k}^{(2)}(x=L)\right] } \\
& /\left[m_{k}^{(2)}(x=0)+m_{k}^{(2)}(x=L)\right] .
\end{aligned}
$$

A positive (negative) value of $S$ corresponds to wave localization at the film surface $x=0(x=L) . S$ is an odd function of $k$ which shows that this mode possesses modeprofile nonreciprocity. Far enough from the hybridization point, the asymmetry parameter $S$ can be shown to scale as $\omega_{M} Q_{01} /\left(\omega_{00}-\omega_{11}\right)$ [18]. Given that $\omega_{00}$ is close to $\omega_{0}$ and $\omega_{11}$ to $\omega_{1}$, this term is very close to the factor $\omega_{M} Q_{01} /\left(\omega_{0}-\omega_{1}\right)$, which enters Eq. (21). This confirms that the origin of the OFIFS is the modal-profile nonreciprocity, in agreement with the naive picture in Fig. 5 and the exchangefree theory in the Appendix. From the comparison of the scaling law for the modal-profile asymmetry and Eq. (21) one finds that the positive $\delta \omega$ corresponds to the anomalous wave localization, i.e., localization of the wave at the surface opposite to one at which the exchange-free DE wave is localized. Similarly, the negative $\delta \omega$ corresponds to the normal localization [Eq. (2)]. The calculated profiles for the dipole-
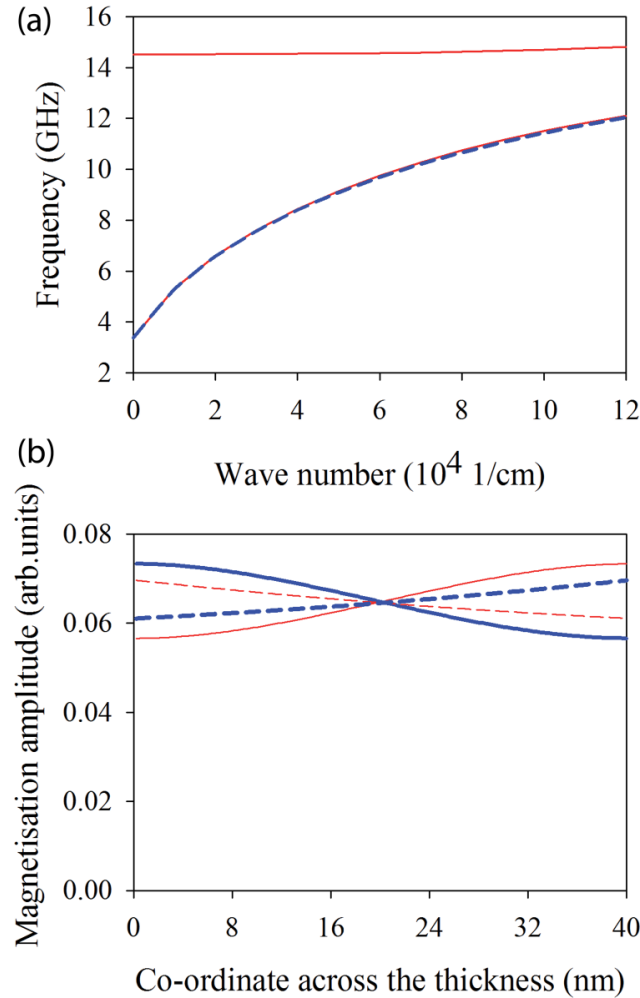

FIG. 6. (Color online) (a) Dispersion of dipole-exchange spin waves in a 40-nm-thick permalloy film for $I=0$. Parameters of calculation. Film thickness $40 \mathrm{~nm}$, saturation magnetization $4 \pi M=$ $10500 \mathrm{G}$, gyromagnetic coefficient $\gamma / 2 \pi=2.8 \mathrm{MHz} / \mathrm{Oe}$, internal static magnetic field is $137.2 \mathrm{Oe}$, and exchange constant $A=1.355 \times$ $10^{6} \mathrm{erg} / \mathrm{cm}\left(\alpha=3.04 \times 10^{-13} \mathrm{~cm}^{2}\right)$. Dashed line: Exchange-free Damon-Eshbach dispersion law (given here for comparison). (b) Modal profiles of the fundamental mode of the dipole-exchange waves (solid lines) and of exchange-free Damon-Eshbach waves (dashed lines). Thick lines $k>0$, and thin lines $k<0$.

exchange waves are shown in Fig. 6(b). For comparison, the respective modal profiles calculated with the exchange-free theory are also displayed in this figure.

The dependence of the OFIFS on the wave number is quite steep. If one expands the product $Q_{01}\left(P_{00}-P_{11}\right)$ in the numerator of Eq. (21) into Taylor series in $k$, one obtains that for $k L \ll 1$ the leading term of the expansion is the $(k L)^{2}$ term. The leading term of the Taylor expansion of the denominator is $k^{0}$ one. Thus, the dependence of the OFIFS is at least parabolic.

\section{Numerical results}

The result of our calculation by using Eq. (21) is shown in Fig. 7 for a current density of $6.67 \times 10^{6} \mathrm{~A} / \mathrm{cm}^{2}$. We perform this calculation for the parameters of the film we use in our experiment (solid line, see the figure caption for the details). The Oersted field induced frequency shift grows quite quickly with an increase in the wave number. Given the scaling law above $\left[(k L)^{2}\right.$ for very small $k$ and steeper for larger $k$ values], this suggests that for thick films OFIFS may become dominating for large wave numbers, since the Doppler frequency shift $\Delta f_{\text {Dop }}$ scales linearly with $k$ and is independent from $L$. In the same figure we also show the 


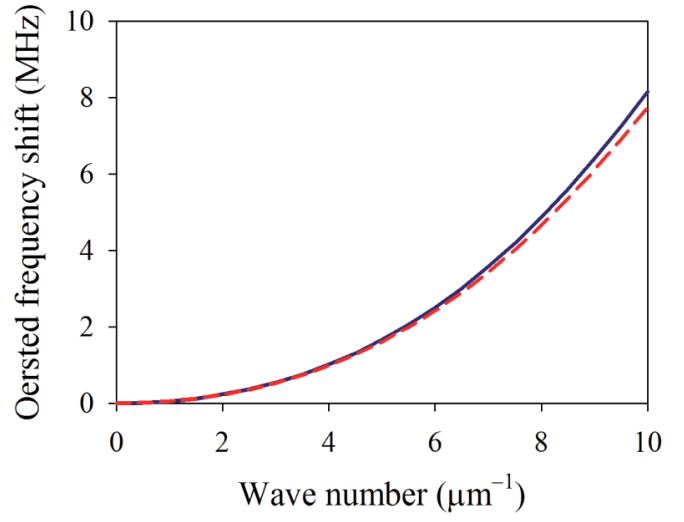

FIG. 7. (Color online) Calculated Oersted field induced frequency shift for dipole exchange spin waves for a current density $J=6.67 \times 10^{6} \mathrm{~A} / \mathrm{cm}^{2}$. Solid line: Internal field is $137.2 \mathrm{Oe}$. Dashed line: Internal field is 280 Oe. Other parameters of calculation are the same as in Fig. 6. Equation (21) was used to produce these data

result of the calculation for a larger internal field (280 Oe, dashed line). One sees that the applied field does not have a significant effect on the magnitude of the OFIFS.

The dash and dot-dash curves in Fig. 4 are the calculated total frequency shift $\Delta f_{\text {tot }}=\Delta f_{\text {Dop }}+\delta f_{\text {Oe }}$ for for the 40 and 10 -nm-thick films assuming a degree of spin polarization of the current $P=0.6$. The other parameters of calculation are the same as for Fig. 6. Here $\delta f_{\text {Oe }}$ is deduced from Eq. (21) as $\delta f_{\text {Oe }}=-\delta \omega /(2 \pi)$, to comply with the sign convention used in the definition of $\delta f_{\text {odd }}$. From this figure one sees that the dependence deviates from the linear one with a negative slope starting from very small wave numbers and the total shift becomes negative for $k>6 \mu \mathrm{m}^{-1}$. Starting from this $k$ value the OFIFS represents the dominating contribution to the total frequency shift.

We also calculate the characteristic value of $k$ for which the OFIFS starts to provide a contribution to the total frequency shift of a specific magnitude. We consider two cases: when $\delta f_{\text {Oe }}$ becomes either $5 \%$ or $10 \%$ of the Doppler shift. These data are shown in Fig. 8. From this figure one sees that the maximum $k$ value, for which the contribution of OFIFS to the measured degree of spin polarization can be regarded as negligible to experimental accuracy, drops very quickly with the film thickness. This is due to the above discussed steep dependence of $\delta \omega$ on $L$. This characteristic wave number also depends on the applied field through the dependence of the mode frequencies in the denominator of Eq. (21) on the applied field. However, the dependence is not very strong which is seen from the comparison of two plots in this figure: for $H=$ 137.2 Oe and $5 \mathrm{kOe}$.

In this graph we also compare two competing methods of extracting the spin polarization from the Doppler shift data: Ours which is based on the measurement of the frequency and the one from Ref. [9] which is based on measurement of the spin-wave group velocity. One sees that results of the measurements of the variation in the group velocity due to the presence of a dc current should be much stronger affected by OFIFS than the measurements of the frequency for the same value of $k$. Indeed, the characteristic $k$ for the group velocity measurements allowing a $10 \%$ contribution of OFIFS

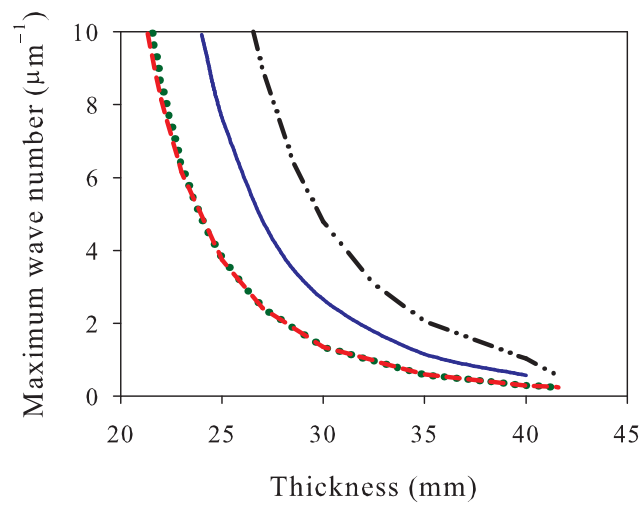

FIG. 8. (Color online) Characteristic value of the spin-wave number for which the Oersted field induced frequency shift starts to give contribution to the total frequency shift of particular magnitude. Solid line: $\delta f_{\text {Oe }} / \Delta f_{\text {Dop }}=10 \%$, internal field is 137.2 Oe; dashed line: $\delta f_{\text {Oe }} / \Delta f_{\text {Dop }}=5 \%$, internal field is 137.2 Oe; dash-dotted line: $\delta f_{\text {Oe }} / \Delta f_{\text {Dop }}=10 \%$, internal field is $5 \mathrm{kOe}$. Dotted line: Approach from Ref. [9] of using the difference of group velocities instead of the frequency difference to extract the degree of spin polarization. Internal field is $137.2 \mathrm{Oe}$ and the assumed contribution of the Oersted field induced frequency shift to the total frequency shift is $10 \%$. (Note that occasionally this line almost overlaps with the dashed line.) The Doppler shift is calculated for $P=0.6$. All other parameters of calculation are the same as for Fig. 6. Equation (21) was used to produce these data.

to the total frequency shift coincides with the characteristic $k$ value for the $5 \%$ contribution of OFIFS in the frequency measurements.

In this section we also consider another effect which has not been taken into account yet in our theory. This is the nonreciprocity of spin-wave dispersion seen for the 40-nm-thick sample for $I=0$ (see Fig. 2). This type of nonreciprocity is usually attributed to the nonuniformity of material parameters across the film thickness [16]. One of these potential nonuniformities is surface magnetization pinning which originates from the presence of surface anisotropy. Another possible reason which is worth mentioning in this context is spontaneous formation of a thin surface sublayer whose magnetic properties are different from the bulk of the material (see, e.g., the discussion in Ref. [27]). Here for simplicity we assume the presence of magnetization pinning at one of the film surfaces. To understand the effect of the single-side pinning we perform direct numerical solution of Eq. (6). To obtain the effect of the nonreciprocity, we assume that spins at the film surface facing the antennae are partly pinned and are completely unpinned on the other film surface. This simulation gives the correct values for the spin-wave frequencies. For instance, one obtains the same value as in the experiment $(10.709 \mathrm{GHz})$ for $k_{z}=+7.8 \mu \mathrm{m}^{-1}$ and $I=10 \mathrm{~mA}$. For the wave propagating in the opposite direction both theory and experiment give $10.749 \mathrm{GHz}$. The value of the surface pinning parameter used in this calculation is $1.8 \times 10^{5} \mathrm{~cm}^{-1}$. This value corresponds to the value of the constant of the surface normal uniaxial anisotropy of $0.245 \mathrm{~mJ} / \mathrm{m}^{2}$. 
The solid line in Fig. 4(b) is actually the total currentinduced frequency shift extracted from this numerical calculation, taking into account a partial pinning of the magnetization at one film surface. As in the experiment we calculate and show $\delta f_{\text {odd }}$ in Fig. 4, in order to remove the even contribution to the total frequency shift from the raw simulation data (as discussed in Sec. II B, an even contribution originates from the interplay between the Oersted field and an asymmetric surface pinning). The dashed line in Fig. 4(b) is the result of our analytical solution [Eq. (21)]. This solution assumes unpinned surface spins at both film surfaces and, consequently, no frequency nonreciprocity in the absence of the dc current. The very good agreement of the dashed line with the solid one confirms the validity of our experimental approach for removing the $I=0$ nonreciprocity from the experimental data by calculating $\delta f_{\text {odd }}$. The dots in the figure are the available five experimental points. One sees good quantitative agreement with the experiment.

The last point which we want to comment on in this section is the effect of the finite strip width. Our numerical solutions of a 2D version of Eq. (6) shows that the presence of the geometrical confinement in the plane of the film does not change the OFIFS qualitatively. The dominating effect of the confinement is a frequency shift for $I=0$ due to the static demagnetizing field which appears because the strip is magnetized along a hard axis. This effect is easily taken into account in our 1D model above of an "effective" continuous film by subtracting some effective demagnetizing field $H_{\text {dem }}$ from the applied field in Eq. (18). In the example of Fig. 2 we subtract 142.8 Oe from the applied field in order to obtain the good agreement with the experiment. This value is quite close to the value of the static demagnetizing field averaged across the area of the stripe cross section which we obtain with the LLG Micromagnetic Simulator [38] for the applied field of 280 Oe in this geometry.

\section{CONCLUSION}

In this paper we have studied the current-induced frequency shift for spin waves propagating perpendicular to the direction of the applied field in an in-plane magnetized 40-nm-thick permalloy strip. Contrary to the previous measurements of current-induced spin-wave Doppler shift in thinner films, this experiment revealed a nonmonotonic dependence of the extracted degree of spin polarization on the spin-wave number. For large wave numbers, the extracted value of the degree of spin polarization is negative, which is unphysical. We suggest that this phenomenon originates from a contribution from a concurrent effect, namely a spin-wave frequency nonreciprocity induced by the Oersted field generated by the dc current applied to the sample in order to observe the Doppler effect. This contribution to the total frequency shift is experimentally indistinguishable from the Doppler frequency shift and grows with an increase in the sample thickness.

To confirm this idea, a theory of the Oersted field induced nonreciprocal frequency shift has been constructed. The theory unambiguously demonstrates the dominating role of this type of frequency nonreciprocity in the formation of the total frequency shift in the presence of a dc current for permalloy films with thicknesses above $20 \mathrm{~nm}$. The comparison of the theory with the experiment also confirms the recent theoretical prediction of the anomalous modal-profile nonreciprocity for large-magnetic-moment metallic ferromagnetic films [18].

This work allows one to understand the limitations of the technique of the current-induced spin-wave Doppler shift when carried out in the magnetostatic surface wave (or DamonEshbach) geometry. We found that this configuration is fully appropriate for film thickness $20 \mathrm{~nm}$ or below. For thicker films care should be taken in order to avoid the situation where the effect of the Oersted field potentially becomes dominant. As follows from our theory, using small wave vectors is a way to avoid it.

The present case of the 40-nm-thick film and large $k$ values is a clear example of such unfavorable experimental conditions. Even in this situation, the current-induced modification of spin-wave propagation can be measured very precisely and interpreted with an explicit analytical theory. In our opinion, this possibility is a natural advantage of using spin waves for probing the spin-transfer torque. Indeed, due to the simple plane-wave structure and linearity of small-signal spin waves, accurate explicit analytical models can be constructed in 2D (and simple numerical algorithms can be developed in 3D). This is in strong contrast to the more widely studied case of the current-induced domain-wall motion. Since the domain walls are intrinsically nonlinear objects, full (nonlinear) micromagnetic models are required, even in the simplest cases. For instance, full 3D micromagnetic simulations are necessary to treat the influence of the same effect of the Oersted field on the domain wall dynamics.

\section{ACKNOWLEDGMENTS}

This work was supported by the ANR (NanoSWITI, ANR11-BS10-003) and the Australian Research Council.

\section{APPENDIX: EXCHANGE-FREE THEORY OF THE OERSTED FIELD INDUCED NONRECIPROCAL FREQUENCY SHIFT FOR MAGNETOSTATIC SURFACE WAVES}

The exchange-free theory for the magnetostatic surface waves was first suggested by Damon and Eshbach [14] more than 50 years ago. The straightforward way to obtain this result is by solving the second-order partial derivative equation-called Walker equation [30]—employing the appropriate electrodynamic boundary conditions. (Walker equation is derived by solving the Landau-Lifshitz equation for the magnetic torque together with Maxwell equations in the magnetostatic approximation.) Although the Walker-equation approach is the standard way to tackle the spin-wave dispersion problem, in this paper we will follow a different route: We will solve the eigenvalue problem for the same system of integral equations as used in Sec. IV to develop the dipole-exchange theory of OFIFS [Eqs. (6)-(8)]. An exact analytical solution exists for this system for the vanishing dc current $\mathbf{I}$ and $\alpha=0$. We will employ this solution as the zero approximation to construct the perturbation theory for nonvanishing values of I. The Green's function approach makes the perturbation theory especially simple. 
The analytical solution of the system (6)-(8) for $\alpha=0$ and $\mathbf{I}=0$ has the form

$$
\mathbf{m}_{k}=\left|\begin{array}{c}
m_{k}^{(1)} \\
m_{k}^{(2)}
\end{array}\right|=\left|\begin{array}{c}
B \exp (-k x) \\
-A \exp (k x-L)
\end{array}\right|
$$

After substitution of this solution in Eq. (6) and some straightforward algebra one finds that the two eigenvalues $\omega_{1}=+\omega_{0}$ and $\omega_{2}=-\omega_{0}\left(\omega_{0}>0\right)$ of the system of the integral equations (6)-(8) are given by the Damon-Eshbach dispersion relation [14]

$$
\omega_{0}^{2}=\omega_{H}\left(\omega_{H}+\omega_{M}\right)+\frac{\omega_{M}^{2}}{4}[1-\exp (-2 k L)] .
$$

The respective right-hand (column) eigenvectors are as follows:

$$
\begin{aligned}
& \left|\mathbf{m}_{1}\right\rangle=\left|\begin{array}{c}
\exp (-k x) \\
-\frac{2 \omega_{H}-2 \omega_{0}+\omega_{M}}{\omega_{M}} \exp (k x)
\end{array}\right|, \\
& \left|\mathbf{m}_{2}\right\rangle=\left|\begin{array}{c}
\exp (-k x) \\
-\frac{2 \omega_{H}+2 \omega_{0}+\omega_{M}}{\omega_{M}} \exp (k x)
\end{array}\right| .
\end{aligned}
$$

From Eq. (3) it follows that for a given $k>0$ the eigenwave with the positive frequency $\omega_{1}$ propagates in the positive direction of the axis $z$ and the wave with the negative eigenfrequency $\omega_{2}$ travels in the opposite direction. In the following we will use this convention (a positive or negative $\omega$ for an always positive $k$ ) to identify the waves traveling in particular directions along the axis $z$. This way is natural, given the role of $\omega$ as an eigenvalue of Eq. (6).

The surface character of the waves follows from Eqs. (A3) and (A4). From Eq. (A2) it follows that $\omega_{0}$ never exceeds the limiting value $\omega_{H}+\omega_{M} / 2$. Therefore one finds that $\frac{2 \omega_{H}+2 \omega_{0}+\omega_{M}}{\omega_{M}} \exp (k x)$ is always larger (and usually significantly larger) than $\exp (-k x)$, and that $\frac{2 \omega_{H}-2 \omega_{0}+\omega_{M}}{\omega_{M}} \exp (k x)$ is (significantly) smaller than $\exp (-k x)$. Thus, the wave with the positive frequency is localized near the lower film surface $x=$ 0 [Eq. (A3)] and the wave with the negative eigenfrequency is localized at the upper film surface $x=L$ [Eq. (A4)].

For the further analysis we will also need the respective left-hand eigenvectors. (Since the integral operator of this equation is not symmetric, the right-hand eigenvectors are not orthogonal to each other but are orthogonal to the respective left-hand eigenvectors.) The left-hand (row) eigenvectors are given by the following equations:

$$
\left\langle\mathbf{m}_{1}|=| \frac{2 \omega_{H}+2 \omega_{0}+\omega_{M}}{\omega_{M}} \exp [k(L-x)] ; \exp [k(x-L)]\right|,
$$

$$
\left\langle\mathbf{m}_{2}|=| \frac{2 \omega_{H}-2 \omega_{0}+\omega_{M}}{\omega_{M}} \exp [k(L-x)] ; \exp [k(x-L)]\right| .
$$

One can easily see that these sets of vector functions are orthogonal: $\int_{0}^{L}\left\langle\mathbf{m}_{i}(x) \mid \mathbf{m}_{j}(x)\right\rangle d x=0$ for $i \neq j$.

Now we introduce the Oersted field $H_{\mathrm{Oe}}$ of a dc current I with a current density $\mathbf{J}$ flowing along the axis $z$. This field has only one component $H_{\mathrm{Oe} y}=H_{\mathrm{Oe}}=J_{0} J(x-L / 2)$, which is antisymmetric with respect to the half-thickness of the film $(x=L / 2$, see Fig. 5). This field combines with the applied field $\mathbf{H}$ giving rise to the term $\omega_{H}+\gamma H_{\mathrm{Oe}}$ in Eq. (6). Since $H \gg H_{\mathrm{Oe}}$ we may treat $\gamma H_{\mathrm{Oe}}$ as a perturbation term for the $\mathbf{I}=0$ equation [i.e., Eq. (6) with $H_{\mathrm{Oe}}=0$ ]. This operator perturbation gives rise to a perturbation of the eigenvalues of the original operator, i.e., to an eigenfrequency shift $\delta \omega(I)=\omega(I)-\omega(I=0)$. In the first approximation this frequency shift reads

$$
\delta \omega_{i}=\int_{0}^{L}\left\langle\mathbf{m}_{i}(x)|\delta C(x)| \mathbf{m}_{i}(x)\right\rangle d x / \int_{0}^{L}\left\langle\mathbf{m}_{i}(x) \mid \mathbf{m}_{i}(x)\right\rangle d x,
$$

where $i=1,2$ indexes the unperturbed eigenvalues and eigenfunctions [see Eqs. (A3) and (A4)] and $\delta C(x)$ is the operator of the perturbation:

$$
\delta C(x)=\gamma J J_{0}(x-L / 2)\left|\begin{array}{cc}
-1 & 0 \\
0 & 1
\end{array}\right| .
$$

Calculation of the integrals in Eq. (A7) reveals that $\delta \omega$ is even in frequency:

$\delta \omega_{2}(I)=\delta \omega_{1}(I)=\delta \omega(I)=\gamma J J_{0}[1-k L \quad \operatorname{coth}(k L)] /(2 k)$.

As a result, the total frequency shift due to the Oersted field reads

$$
\begin{aligned}
\delta \omega_{\mathrm{Oe}} & =\frac{\omega_{1}+\delta \omega(I)-\left|\omega_{2}+\delta \omega(I)\right|}{2} \\
& =\delta \omega(I)=\gamma J J_{0}[1-k L \quad \operatorname{coth}(k L)] /(2 k) .
\end{aligned}
$$

Equation (A7) demonstrates the important role of the wave profile nonreciprocity in the formation of the Oersted frequency shift. Indeed, the magnitude of the shift is given by the projection of the thickness profile of the perturbation (which is antisymmetric in $x-L / 2$ ) on the basis of the modal profiles of the eigenwaves given by Eqs. (A3) and (A4). These profiles are completely uniform (symmetric) across the film thickness for $k=0$. As a result, $\delta \omega_{\text {Oe }}=0$ for $k=0$. With an increase in $k$ the frequency shift scales as

$$
\delta \omega_{\mathrm{Oe}} \simeq-\gamma J J_{0} L^{2} k\left[1-(k L)^{2} / 15\right] / 6 .
$$

Thus, its magnitude grows with an increase in $k$ in a way similar to the increase in the surface character of the waves with the increase in $k$.

As already mentioned in the text, the frequency shift given by Eq. (A10) cannot explain our data, basically because its sign is the same as that of the Doppler shift, so that it cannot explain the behavior observed in Fig. 4(b). In our understanding, this is strong experimental evidence for the failure of the Damon-Eshbach theory for describing the mode profiles in this case, although it happens to predict very correctly the mode dispersion [see Figs. 6(a) and 6(b)]. 
[1] V. V. Kruglyak, S. O. Demokritov, and D. Grundler, J. Phys. D: Appl. Phys. 43, 260301 (2010).

[2] R. Hertel, W. Wulfhekel, and J. Kirschner, Phys. Rev. Lett. 93, 257202 (2004).

[3] M. P. Kostylev, A. A. Serga, T. Schneider, B. Leven, and B. Hillebrands, Appl. Phys. Lett. 87, 153501 (2005).

[4] A. Khitun, M. Bao, and K. L. Wang, IEEE Trans. Mag. 44, 2141 (2008).

[5] V. Vlaminck and M. Bailleul, Science 322, 410 (2008).

[6] M. Zhu, C. L. Dennis, and R. D. McMichael, Phys. Rev. B 81, 140407(R) (2010).

[7] M. Zhu, B. D. Soe, R. D. McMichael, M. Carey, S. Maat, and J. Childress, Appl. Phys. Lett. 98, 072510 (2011).

[8] R. L. Thomas, M. Zhu, C. L. Dennis, V. Misra, and R. D. McMichael, J. Appl. Phys. 110, 033902 (2011).

[9] K. Sekiguchi, K. Yamada, S. M. Seo, K. J. Lee, D. Chiba, K. Kobayashi, and T. Ono, Phys. Rev. Lett. 108, 017203 (2012).

[10] M. Haidar and M. Bailleul, Phys. Rev. B 88, 054417 (2013).

[11] J.-Y. Chauleau, H. G. Bauer, H. S. Körner, J. Stigloher, M. Härtinger, G. Woltersdorf, and C. H. Back, Phys. Rev. B 89, 020403 (2014).

[12] S. M. Seo, K. J. Lee, H. Yang, and T. Ono, Phys. Rev. Lett. 102, 147202 (2009).

[13] D. Stancil and A. Prabhakar, Spin Waves Theory and Applications (Springer, Berlin, 2009).

[14] R. Damon and J. Eshbach, J. Phys. Chem. Solids 19, 308 (1961).

[15] T. T. Schneider, A. A. Serga, T. Neumann, B. Hillebrands, and M. P. Kostylev, Phys. Rev. B 77, 214411 (2008).

[16] P. Amiri, B. Rejaei, M. Vroubel, and Y. Zhuang, Appl. Phys. Lett. 91, 062502 (2007).

[17] P. Grünberg, C. Mayra, W. Vacha, and M. Grimsditch, J. Magn. Magn. Mater. 28, 319 (1982).

[18] M. P. Kostylev, J. Appl. Phys. 113, 053907 (2013).

[19] M. Bailleul, D. Olligs, and C. Fermon, Appl. Phys. Lett. 83, 972 (2003).

[20] V. Demidov, M. P. Kostylev, K. Rott, P. Krzysteczko, G. Reiss, and S. Demokritov, Appl. Phys. Lett. 95, 112509 (2009).

[21] K. Sekiguchi, K. Yamada, S. M. Seo, K. J. Lee, D. Chiba, K. Kobayashi, and T. Ono, Appl. Phys. Lett. 97, 022508 (2010).

[22] V. Vlaminck and M. Bailleul, Phys. Rev. B 81, 014425 (2010).
[23] M. Haidar, Ph.D. thesis, Université de Strasbourg, 2012.

[24] P. R. Emtage, J. Appl. Phys. 49, 4475 (1978).

[25] B. Hillebrands, Phys. Rev. B 41, 530 (1990).

[26] M. Vohl, J. Barnas, and P. Grunberg, Phys. Rev. B 39, 12003 (1989).

[27] M. Kostylev, A. A. Stashkevich, A. O. Adeyeye, C. Shakespeare, N. Kostylev, K. Ross, N. Kennewell, R. Magaraggia, Y. Roussigné, and R. L. Stamps, J. Appl. Phys. 108, 103914 (2010).

[28] Comparing Figs. 2(a) and 2(b), one notices a global frequency shift of about $70 \mathrm{MHz}$. For the main peak, the $(k<0,+H)$ curves intersect the horizontal axis around $8.05 \mathrm{GHz}$, while the $(k>0,-H)$ curve intersect it around $7.98 \mathrm{GHz}$. The same effect is observed more clearly in Fig. 3. This large shift is not related to any MSSW nonreciprocity, but rather to an uncertainty in the magnitude of the applied field of the order of $1 \mathrm{mT}$ (our probe station cannot be equipped with a Hall probe). This uncertainty does not affect the current-induced frequency shifts $\delta f_{i j}$ defined in Sec. II B, since they are obtained by comparing signals measured for the same field polarity.

[29] J. Banhart, H. Ebert, and A. Vernes, Phys. Rev. B 56, 10165 (1997).

[30] A. Gurevich and G. Melkov, Magnetization Oscillations and Waves (CRC, Boca Raton, FL, 1996).

[31] For example, for $M / / e_{y}$, Eq. (2) predicts that the $-k$ wave is localized close to the top surface, whereas it was assumed in Fig. 5(a) that it is localized close to the bottom surface.

[32] B. A. Kalinikos, Sov. J. Phys. 24, 718 (1981).

[33] S. G. Mikhlin, Variational Methods in Mathematical Physics (Pergamon, New York, 1964).

[34] R. E. De Wames and T. Wolfram, J. Appl. Phys. 41, 987 (1970).

[35] More precisely, the $n$th SSW branch enters the spectrum of the DE wave for the "critical" thickness $L_{n}^{2}=$ $n^{2} \alpha \pi^{2} /\left(\sqrt{v^{2}+v+0.5}-v-0.5\right)$, where $v=H /\left(4 \pi M_{s}\right)$. In our experiment $v=0.028$ and $\alpha=3.1 \times 10^{-13}$, which gives $L_{1}=39 \mathrm{~nm}$ and $L_{2}=156 \mathrm{~nm}$.

[36] B. Kalinikos and A. Slavin, Solid State Phys. 19, 7013 (1986).

[37] This expression of the OFIFS is for CGS units ( $L$ should be taken in $\mathrm{cm}$ and $\mathrm{J}$ in $\mathrm{A} / \mathrm{cm}^{2}$ ). The same formula can be used in SI units provided $J_{0}$ is replaced by $\mu_{0}$.

[38] M. Scheinfein, LLG micromagnetic simulator, http://llgmicro. home.mindspring.com, 2009. 